\title{
Rational Power Supply of Energy-Deficient Regions
}

\author{
Anton Verteshev \\ Faculty of Computer Science and \\ Electrical Power Engineering \\ Pskov State University \\ Pskov, Russia \\ a_verteshev@mail.ru \\ Dmitry Andreev \\ Faculty of Computer Science and \\ Electrical Power Engineering \\ Pskov State University \\ Pskov, Russia \\ dandreev60@mail.ru
}

Abstract-The questions of supplying power to the region with remote consumers are considered. The model of integrated power supply is proposed. Mathematical dependences that allow accounting volumes of certain types energy resources consumption and forming rational energy balance of the region are formulated.

Keywords-energy balance of the region, centralized power supply, electricity consumers, decentralized power supply.

\section{INTRODUCTION}

Efficient power supply of energy-deficient regions (remote consumers of electricity) determines the conditions for safe, efficient and sustainable functioning of the energy sector, as well as the system of relations between the subjects of the fuel and energy complex, consumers and the state, ensuring the achievement of the goals set in energy policy for the real conditions of the country's energy sector [1].

The strategic goals of supplying power to the energydeficient regions are:

1) Reduction of consumption of non-renewable fuel and energy resources (fuel and energy resources);

2) Reducing the environmental impact from the fuel and energy infrastructure;

3) Improving economic efficiency of supplying energy to a region;

4) Ensuring reliable power supply for geographically distant consumers.

From these goals we can suggest following ways to achieve them:

a) Integrated use of centralized and decentralized energy supply for large enterprises and remote energy-deficient regions;

b) Quick creation of autonomous (decentralized) facilities (systems) based on unconventional and renewable energy sources;

c) Introduction of elements of intelligent energy systems (IES) for monitoring and control, transmission and distribution of electricity, as well as the effective management of electrical equipment at enterprises and utilities [2].

\section{MODEL OF INTEGRATED POWER SUPPLY}

Generalized scheme of the energy balance of the region (Pskov region) is shown in "Fig. 1".

Assuming that in the region there are no other consumers rather than enterprises, designated as "K", it is possible to write the following formula:

$$
\sum_{i=1}^{I} W_{i}^{c}+\sum_{\kappa=1}^{K} W_{\kappa}^{d}=\sum_{\kappa=1}^{K} W_{\kappa}\left(1+\alpha_{\kappa}\right)
$$

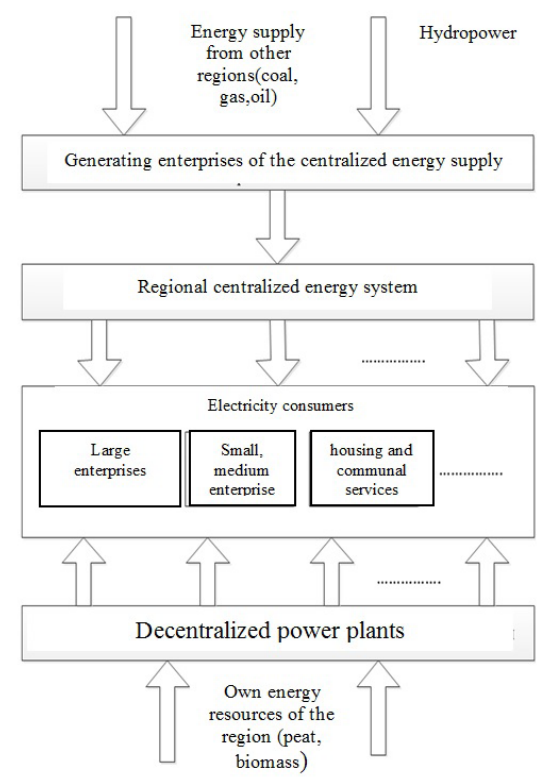

Fig. 1. Generalized scheme of the energy balance of some region, the Pskov region, for example.

Print ISSN 1691-5402

Online ISSN 2256-070X

http://dx.doi.org/10.17770/etr2019vol1.4139

(C) 2019 Anton Verteshev, Sergey Verteshev, Dmitry Andreev, Olga Timofeeva.

Published by Rezekne Academy of Technologies.

This is an open access article under the Creative Commons Attribution 4.0 International License. 
Let's introduce following notation:

where: $W_{i}^{c}$ - is electrical energy generated by the $\mathrm{i}$-th enterprise $(\mathrm{i}=1 \ldots \mathrm{I})$, that provides the operation of a centralized power supply system

$W_{\hat{e}}^{d}$ - is electrical energy generated by an autonomous (decentralized) source;

$W_{\kappa}$ - is energy consumed at the K-th enterprise

$\left(\mathrm{K}=1 \ldots \ldots .(\mathrm{K}) ;\right.$ coefficient $\quad W_{k}$, that takes into account losses $W_{\kappa}$ during transportation of electricity from the source to the consumer [3];

$W_{\kappa}=W_{k(\text { original })}-W_{k(\text { losses })}$, where $W_{k(\text { losses })}$ is energy losses during transportation.

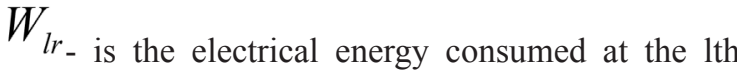
enterprise $(1=1 \ldots)$ in the $r$ th area $(r=1 \ldots . R)$, where $\mathrm{R}$ is the number of areas in the subject of the Russian Federation (for the Pskov region, $\mathrm{R}=26$ );

$W_{n r_{-}}$is electric energy consumed by housing and utilities enterprises and individual users from the population in the $\mathrm{r}$-th area $\left(\mathrm{n}=1 \ldots N_{r}\right) ; \mathrm{M}-$ is a total number of autonomous (decentralized) sources of electrical energy in the region( $M \leq K+\sum_{r=1}^{R}\left(L_{r}+N_{r}\right)$ ,autonomous (decentralized) sources that consumers can have, but in practice they do not. For the case when there is a transfer to a centralized network of excess electricity generated by an Autonomous source, the ratio will take form:

$$
\sum_{i=1}^{I} W_{i}^{c}+\left(\sum_{\kappa=1}^{K} W_{\kappa}^{d}-W_{\text {excess }}{ }^{d}\right)=\sum_{\kappa=1}^{K} W_{\kappa}\left(1+\alpha_{\kappa}\right)
$$

The energy balance of the region can be written in the following form:

$$
\begin{aligned}
& \sum_{i=1}^{I} W_{i}^{c}+\sum_{m=1}^{M} W_{m}^{d}=\sum_{\kappa=1}^{K} W_{\kappa}\left(1+\alpha_{\kappa}\right) \\
& +\sum_{r=1}^{R}\left[\sum_{i=1}^{L_{r}} W_{l r}\left(1+\alpha_{l r}\right)+\sum_{n=1}^{N_{r}} W_{n r}\left(1+\alpha_{n r}\right)\right]
\end{aligned}
$$

The model of the energy system of the region can be represented by a directed graph, the vertices of which correspond to the sources and consumers, and the edges reflect the direction of electricity transmission ("Fig. 2").

Energy losses during transportation are shown here as the corresponding vertices of the graph ( ). Other notation:

$W_{\alpha(L 1,1)}$ - losses associated with the transmission of energy to the L-th consumer (medium and small enterprises) in the first area;

$W_{(L 1,1)}$ - is the energy consumed by L-consumer in the first area;

$W_{(L 1,1)}$ - is the energy consumed by L-consumer in the first area;

$W^{d}(L 1,1)$ - is electrical energy generated by an autonomous (decentralized) L-th source in the first area [4];

$W_{\alpha(N), ~}^{S}$

$\alpha(N 1,1)$ - losses associated with the transmission of energy to the $\mathrm{N}$-th consumer (utilities and individual users in the first area;

$W_{(N 1,1)}^{S}$

$W_{(N 1,1)}$ - is the energy consumed by the N-consumer (housing and individual users) in the first area;

$W^{d S}{ }_{(N 1,1)}$ - is electric energy generated by an autonomous (decentralized) source $\mathrm{N}$-th source (housing and utilities and individual users of the population) in the first area [5].

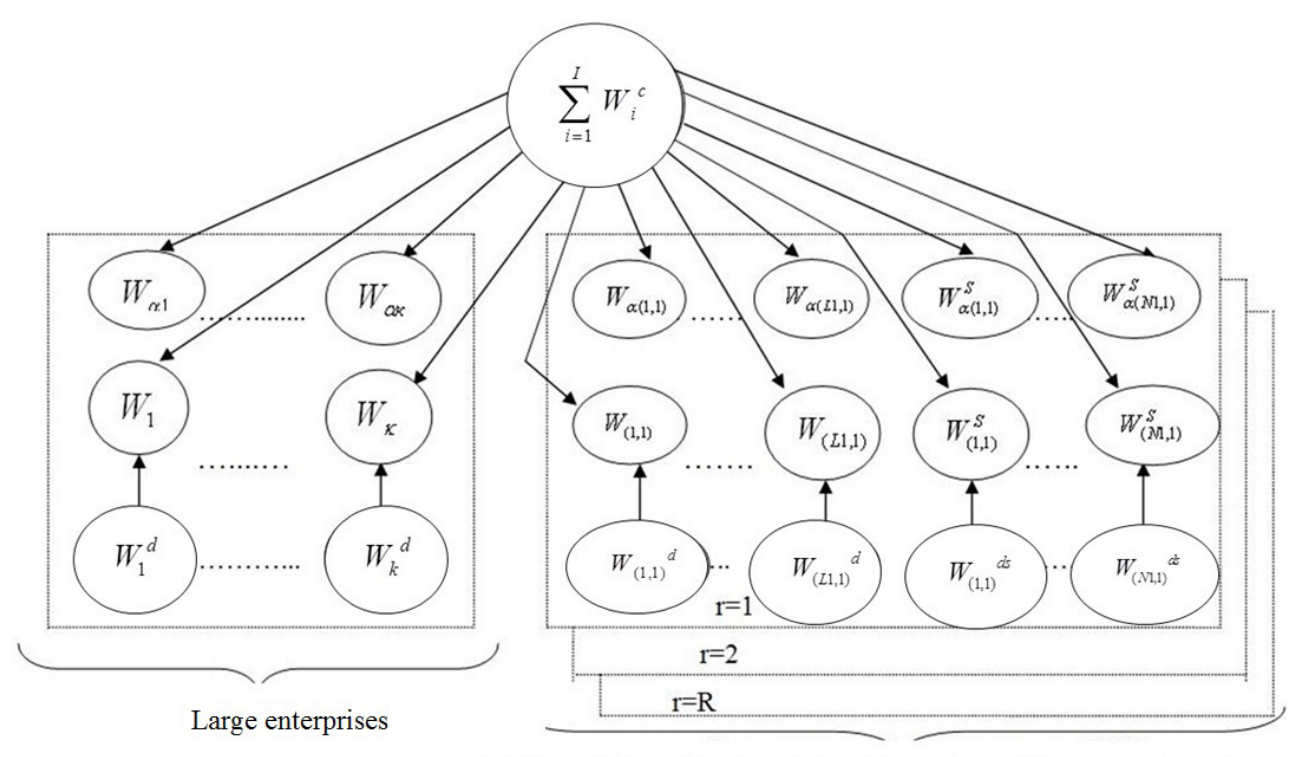

Medium and small enterprises and housing and communal services

Fig. 2. Model of energy supply to consumers of the regional energy system 
III. METHOD OF EVALUATING ECONOMIC EFFECTIVENESS OF THE SYSTEM PROVIDING SUSTAINABLE ENERGY SUPPLY FOR ENERGY DEFICIENT REGION

Let's introduce following notation:

$z^{r e s}$ - is the unit costs associated with the use of fuel and energy resources (coal, gas, etc.), i.e. the cost of fuel and energy resources needed to generate a unit of electricity;

$z^{r e s}=b_{c} \cdot P_{T}$, where $b_{c}$ - specific consumption of fuel and energy resources,

$P_{T}$ - is price of fuel and energy resources; In this case, the absolute costs are equal to:

$z^{\text {res }} \cdot W$, where $W$ - is generated electrical energy;

$z^{\text {exp }}$ - is unit operating costs for the generating plant or a combination of plants of the generating company, required to generate a unit of electrical energy. Absolute costs are: $z^{\exp } \cdot W$, where $W$ - is generated electrical energy.

$z^{c . t r}$ - is specific costs for the transportation of energy from the generating source to the consumer, including losses during transportation per unit of transmitted electrical energy [6].

In this case, the absolute costs are: $z^{\text {c.tr }} \cdot W$, where $\mathrm{W}$ is the consumed electric energy (in this case, we assume that it is equal to the generated electricity).

Costs of centralized power supply: $Z^{c}=\left(z^{c . r e s}+z^{c . \exp }+z^{c . t r}\right) \cdot W$

Costs of integrated use of centralized power supply and energy of Autonomous (decentralized) sources based on non-traditional and renewable energy sources:

$$
\begin{aligned}
& Z^{\text {com }}=\left(z^{d . r e s}+z^{d . \exp }+z^{d . t r}\right) \cdot W^{\prime \prime} \\
& +\left(z^{\text {c.res }}+z^{\text {c.exp }}+z^{c . t r}\right) \cdot W^{\prime}
\end{aligned}
$$

where: $W_{\text {и }}^{\prime \prime} W^{\prime}$ account for electrical energy consumed from a centralized network and from an Autonomous source respectively, $W=W^{\prime \prime}+W^{\prime}$ - is total electrical energy.

Autonomous (decentralized) energy source is usually located directly on consumer's territory. In this regard it can be assumed that the costs of transporting electricity from such a source are negligible: $z^{d . t r} \approx 0$

When using renewable energy sources, the costs for fuel and energy resources can also be considered zero: $z^{\text {d.res }} \approx 0$

In this case, formula (5) takes the form:

$$
\begin{aligned}
& Z^{\text {com }}=\left(z^{d . r e s}+z^{d \cdot \exp }+z^{d . t r}\right) \cdot W^{\prime} \\
& +z^{d \cdot \exp } \cdot W^{\prime \prime}
\end{aligned}
$$

The economic efficiency of using of integrated energy supply is determined by the expression:

$$
\Delta Z=Z^{c}-Z^{c o m}
$$

Taking into account formulas (4) and (6) we get:

$$
\begin{aligned}
& \Delta Z=\left(z^{c . r e s}+z^{c . \exp }+z^{c . t r}\right) \cdot W \\
& -\left(z_{\text {or }}^{\text {d.res }}+z^{d . \exp }+z^{d . t r}\right) \cdot W^{\prime}+z^{d . \exp } \cdot W^{\prime \prime}, \\
& \Delta Z=\left(z^{\text {c.res }}+z^{c . \exp }+z^{c . t r}\right)\left(W-W^{\prime}\right) \\
& -z^{\text {d.exp }} \cdot W^{\prime \prime}
\end{aligned}
$$

A positive economic effect from the integrated energy supply is obtained if:

$$
\left(Z^{\text {c.res }}+Z^{\text {c.exp }}+Z^{\text {c.tr }}\right)-Z^{d . \exp }>0
$$

If the tariff for electricity from the centralized network is overstated comparing to the real costs, this can be taken into account using coefficient $T>1$. In this case, for the consumer the positive economic effect of the integrated energy supply will significantly increase and will be expressed as follows:

$$
\begin{aligned}
& \Delta Z=T \cdot\left(z^{c . r e s}+z^{c . \exp }+z^{c . t r}\right)\left(W-W^{\prime}\right) \\
& -z^{d . \exp } \cdot W^{\prime \prime}
\end{aligned}
$$

Mathematical model reflecting the total cost of integrated energy supply in the region $Z^{\text {reg }}$ and economic efficiency of integrated power supply $\Delta Z^{\text {reg }}$ is defined by following formulas:

$$
\begin{aligned}
& Z^{r e g}=\sum_{\hat{e}=1}^{\hat{E}}\left(z_{\hat{e}}^{c} \cdot W_{k}^{\prime}+z_{k}^{c . t r} \cdot W_{k}^{\prime}+z_{k}^{d} \cdot W_{\hat{e}}^{\prime \prime}\right) \\
& +\sum_{r=1}^{R}\left[\sum_{l=1}^{L_{r}}\left(z_{l r}^{c} \cdot W_{l r}^{\prime}+z_{l r}^{c . t r} \cdot W_{l r}^{\prime}+z_{l r}^{d} \cdot W_{l r}^{\prime \prime}\right)\right. \\
& \left.+\sum_{n=1}^{N_{r}}\left(z_{n r}^{c} \cdot W_{n r}^{\prime}+z_{n r}^{c . t r} \cdot W_{n r}^{\prime}+z_{n r}^{d} \cdot W_{n r}^{\prime \prime}\right)\right] \\
& \Delta Z^{r e g}=\sum_{\hat{e}=1}^{\hat{E}} \Delta Z_{\hat{e}}+\sum_{r=1}^{R}\left[\sum_{l=1}^{L_{r}} \Delta Z_{l, r}+\sum_{n=1}^{N_{r}} \Delta Z_{n, r}\right]
\end{aligned}
$$

where: $z^{c}, z^{d}$ are the unit costs of centralized power supply and costs of energy supply from an autonomous and decentralized sources;

$z^{\text {c.tr }}$ - is the unit costs of transportation of energy from the generating source to the consumer, including losses during transportation per unit of the transmitted electric energy. Thus, absolute cost is: $z^{\text {c.tr }} \cdot W$.

The total cost depends on many variables: the cost of fuel and energy resources ( $Z^{\text {res }}$ ), costs of transportation of electric power taking into account losses $\left(Z^{\text {tr }}\right)$, number and location of large, small and medium-sized consumers (K,L,N, R), etc..[3]

As a criterion for the optimality of the proposed model, the total cost of integrated energy supply in the region can be taken. In this case, the optimality condition takes the form:

$$
Z^{\text {reg }}\left(Z^{\text {res }}, Z^{t r}, K, L, N, R\right) \rightarrow \min .
$$

Other optimality criteria can be used, for example, the 
economic efficiency of integrated energy supply in the region:

$$
\Delta Z^{\text {reg }}\left(Z^{r e s}, Z^{t r}, K, L, N, R\right) \rightarrow \max .
$$

A detailed analysis of the formulas (3), (11) and (12), reflecting the energy balance of the region and the total cost and economic efficiency of integrated energy supply in the region requires taking into account all the parameters characterizing the needs of consumers and the peculiarities of their territorial location in individual areas within their region. For a generalized interpretation of the energy system model of the region, the expression (11) can be presented in the following formulas [7]:

$$
\begin{gathered}
Z^{r e g}=Z^{c}+Z^{c . t r}+Z^{d} \\
Z^{c}=\sum_{\hat{e}=1}^{\hat{E}}\left(z_{\hat{e}}^{c} \cdot P_{k}^{\prime}\right)+\sum_{r=1}^{R}\left(\sum_{l=1}^{L_{r}} z_{l r}^{c} \cdot P_{l r}^{\prime}\right)+\sum_{n=1}^{N_{r}}\left(z_{n r}^{c} \cdot P_{n r}^{\prime}\right)
\end{gathered}
$$

$$
\begin{aligned}
& Z^{c . t r}=\sum_{\hat{e}=1}^{\hat{E}}\left(z_{\hat{e}}^{c . t r} \cdot P_{k}^{\prime}\right) \\
& +\sum_{r=1}^{R}\left(\sum_{l=1}^{L_{r}} z_{l r}^{c . t r} \cdot P_{l r}^{\prime}\right)+\sum_{n=1}^{N_{r}}\left(z_{n r}^{c . t r} \cdot P_{n r}^{\prime}\right) \\
& Z^{d}=\sum_{\hat{e}=1}^{\hat{E}}\left(z_{\hat{e}}^{d} \cdot P_{k}^{\prime \prime}\right) \\
& +\sum_{r=1}^{R}\left(\sum_{l=1}^{L_{r}} z_{l r}^{d} \cdot P_{l r}^{\prime \prime}\right)+\sum_{n=1}^{N_{r}}\left(z_{n r}^{d} \cdot P_{n r}^{\prime \prime}\right)
\end{aligned}
$$

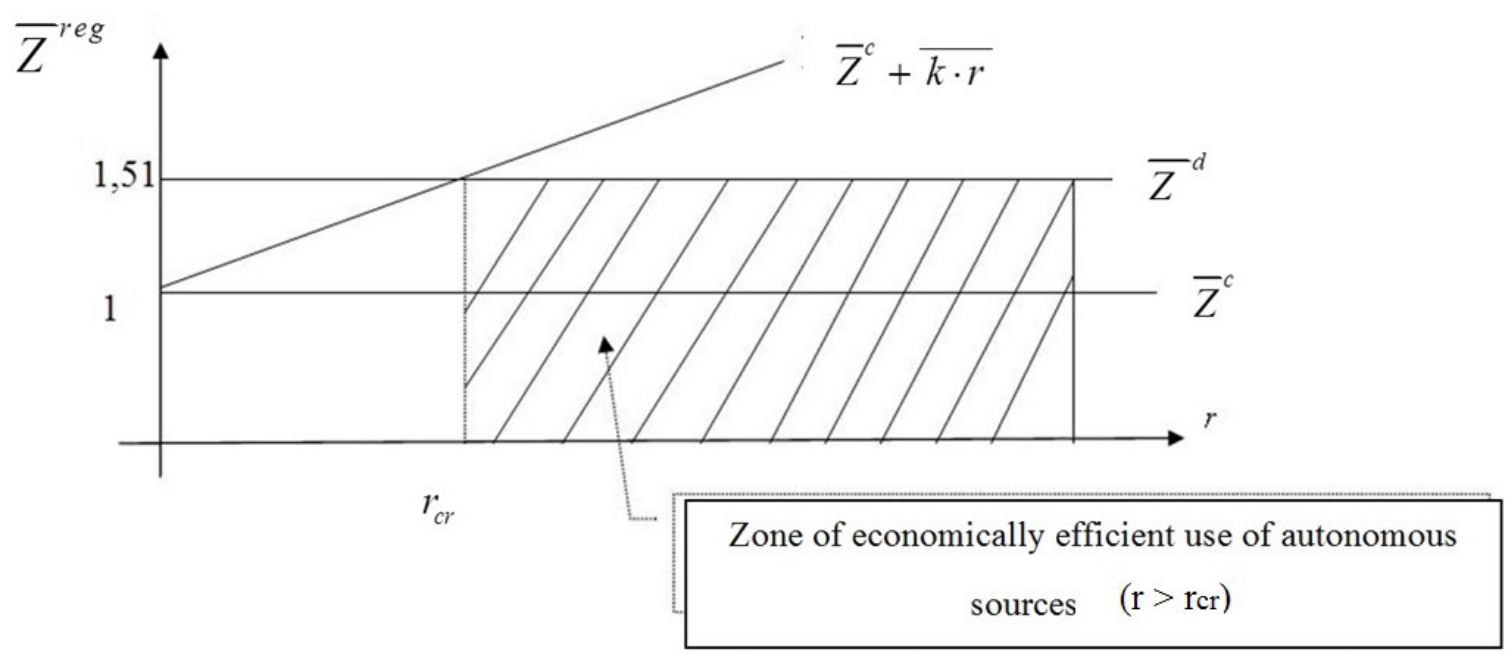

Fig. 3. Dependence of costs vs length of electrical networks for centralized sources and for autonomous energy sources.

If centralized and autonomous sources of electrical energy operate on the same type of fuel and energy resources, the costs of generating electrical energy with an autonomous source are higher. For example, for state district power plant (centralized source) and microturbines operating on natural gas, this ratio is approximately equal to 1.51. Taking into account the above, we will depict in "Fig. 3" components of the formula (20). At length of electric networks in region to $r>r_{c r}$ the use of autonomous energy sources is economically justified (this area is shown in "Fig. 3". indicated by hatching).

For the Pskov region $r_{c r}=\frac{0,51}{0,0005} \approx 1000 \mathrm{~km}$, the total length of electrical networks in the region $r=4608 \mathrm{~km}$.

Assuming that the value of $Z^{\text {c.tr }}$ includes the cost of transmission of electricity, loss of electricity, the cost of maintaining networks and substations, in proportion to the length of the electric networks of the region [8]:

$$
Z^{c . t r}=k \cdot r
$$

where $\mathrm{r}-$ is the length electrical networks; $\mathrm{K}-$ is the coefficient of proportionality.

Based on the established tariffs for energy transmission networks for the region, it is possible to determine the coefficient of proportionality $\mathrm{k}=0,005 \mathrm{RUB} / \mathrm{KWh} \bullet \mathrm{km}$.

Let's move on to the relative variables, taking as a base $Z^{c}$. Denote the relative variables with the upper line formula (15) write in the following form [9]:

$$
\bar{Z}^{r e g}=\bar{Z}^{c}+\overline{k \cdot r}+\bar{Z}^{d}
$$

where $\bar{Z}^{\text {reg }}=\frac{Z^{r e g}}{Z^{c}}, \overline{k \cdot r}=\frac{k \cdot r}{Z^{c}}, \bar{Z}^{d}=\frac{Z^{d}}{Z^{c}}$

If electricity tariffs are too high, and autonomous energy sources use local fuel and energy resources, then the feasibility of using autonomous sources from an economic point of view becomes even more obvious [10]. 


\section{CONCLUSION}

1. The model of integrated power supply is proposed. Formulated mathematical dependences that allow considering volumes of energy resources consumption of each type and forming rational energy balance of the region.

2. The method of assessing the economic efficiency of the system of sustainable energy supply for the energy deficient region and on its basis determined the effectiveness and socio-economic efficiency of the proposed model of energy supply of the Pskov region.

3. Sustainable energy supply for energy-deficient regions is proposed to be formed based on the integrated systems that provide the joint use of centralized and distributed energy supply, including ones, based on non-traditional and renewable energy sources.

\section{REFERENCES}

[1] A. S. Verteshev, "The energy potential of the region and the conditions for its effective use (on the example of Pskov region)," Scientific and technical bulletins of SPbSPU, Economic sciences, No. 1, pp. 62-67, 2012 (in Russian)
[2] A. S. Verteshev and V. R. Okorokov, "Causes and factors determining the possibility and necessity of using non-traditional and renewable energy sources," Proceedings of higher educational institutions, Economics, finance and production management, No. 1, pp. 38-45, 2012. (in Russian)

[3] A. S. Verteshev, ,Distributed energy in ensuring technological safety and economic efficiency of the region," Scientific and technical statements of SPbSPU, Economic sciences, No. 3, pp. 95-100, 2011. (in Russian)

[4] V. Sh. Telyashova and. E. M. Kosmatov, Methods for evaluating the effectiveness and stimulating innovative energy-saving technologies in production and transmission of energy. Saint-Petersburg: Polytechnic University Publishing, 2010. (in Russian)

[5] V. A. Taratin, Strategic planning in energy sector. Saint-Petersburg: Polytechnic University Publishing, 2006. (in Russian)

[6] V. I. Rozova, Economics of industrial energy. Saint-Petersburg: Publisher SPbGPU, 2003. (in Russian)

[7] World Energy Outlook Of Renewable Energy, OECD/IEA, 2018.

[8] World Energy Outlook Of Renewable Energy, OECD/IEA, 2017.

[9] V. R. Okorokov, R. V. Okorokov, and A. S. Verteshev, "Forecast trends of long-term development of the world fuel and energy complex Part 1," Academy of Energy, No. 1, pp. 4-13, 2015. (in Russian)

[10] V. R. Okorokov, R. V. Okorokov, and A. S. Verteshev, "Forecas trends of long-term development of the world fuel and energy complex Part 2," Academy of Energy, No. 2, pp. 4-13, 2015. (in Russian) 